\title{
Départ et accueil : Passer le flambeau
}

\section{P Huston ${ }^{1 \star}$}

Ce fut un plaisir et un privilège d'être rédactrice en chef du Relevé des maladies transmissibles au Canada (RMTC) de 2013 à 2019. Au cours de cette période, la revue a connu une croissance énorme du nombre de lecteurs et d'articles scientifiques, a été remaniée deux fois et s'est dotée de nouvelles fonctionnalités, telles que listes de contrôle des auteurs, communications rapides, infographies et résumés visuels. De plus, le RMTC a été rétabli sur PubMed il y a quelques années. En 2013, le bureau de la rédaction a commencé avec des bureaux, des ordinateurs et quelques anciens numéros de la revue. L'ancien bureau avait fermé ses portes lorsque l'Agence de la santé publique du Canada (ASPC) a été créée en 2004, à la suite du départ à la retraite de l'ancienne rédactrice en chef, Eleanor Paulson. Pendant presque dix ans, il n'y a pas eu d'articles évalués par des pairs et le RMTC a publié principalement des déclarations de comités consultatifs et des résumés de Surveillance de l'influenza. Des appels ont été lancés en faveur du rétablissement de la revue, notamment par le Conseil du réseau de santé publique. Au printemps 2013, le bureau de la revue a été rétabli en tant que projet pilote de deux ans. Grâce à l'appui unanime des centres de la Direction générale de la prévention et du contrôle des maladies infectieuses, le financement permanent de la revue a été assuré en 2014.

Publier une revue, c'est un peu comme élever un enfant - il faut tout un village. Le RMTC est le fruit d'une petite équipe dédiée. Mes sincères remerciements au coordonnatrice à la rédaction, au la résponsable de la production et au conseiller Web qui ont rédigé les numéros mois après mois avec l'aide de nombreux examinateurs, réviseurs, traducteurs, étudiants, d'un graphiste consultant et, de plus en plus, de rédacteurs scientifiques associés. Différents rédacteurs en chef se sont joints à l'équipe dans le cadre d'une affectation de perfectionnement et ont apporté des contributions uniques. Un grand merci au Comité de rédaction du RMTC : au départ, les membres étaient des collègues enthousiastes de l'ASPC, puis, à mesure que le comité grandissait, il est devenu le comité international que nous avons aujourd'hui. Nous avons bénéficié, au fil des ans, des conseils et du soutien incroyables des membres du comité. Le $D^{r}$ John Last, qui est décédé récemment à l'âge de 93 ans, a été un mentor important. Au début des années 1990, il m'a encouragée à devenir rédactrice en chef et a continué d'offrir son amitié et sa sagacité pendant de nombreuses années après son départ officiel à la retraite. L'un des plaisirs que nous avons partagés en tant que rédacteurs en chef a été l'occasion de travailler avec les auteurs pour transformer des manuscrits de l'état de " diamants bruts » en articles que les lecteurs trouvent succincts, instructifs et utiles. J'ai tellement appris dans le cadre de ce travail.

Et maintenant, il est temps de devenir la rédactrice en chef émérite et de passer le flambeau.

J'aimerais souhaiter la bienvenue au $\mathrm{D}^{r}$ Michel Deilgat, à titre de nouveau rédacteur en chef du RMTC. Le Dr Deilgat est un médecin en santé publique qui, après des décennies au service des Forces armées canadiennes en tant que médecin militaire, possède une vaste expérience en médecine opérationnelle, professionnelle et préventive. Il est conseiller médical au Centre des maladies infectieuses d'origine alimentaire, environnementale et zoonotique (CMIOAEZ) de l'ASPC depuis juillet 2011 et a aidé à former une nouvelle génération de professionnels de la santé publique à titre de précepteur du programme de maîtrise en santé publique de I'Université Western. Le Dr Deilgat est titulaire d'une maîtrise en administration publique (École nationale d'administration publique, Université du Québec), d'une maîtrise en enseignement aux professionnels de la santé (M.Ed., Université d'Ottawa) et termine actuellement une maîtrise en sciences de l'information (Université d'Ottawa). Il est membre du comité de rédaction depuis 2014. Je suis persuadée que la revue continuera de s'épanouir grâce aux liens étroits que le Dr Deilgat entretient avec le milieu universitaire et à son engagement envers l'apprentissage continu.

Citation proposée : Huston P. Départ et accueil : Passer le flambeau. Relevé des maladies transmissibles au Canada 2019;45(10):296. https://doi.org/10.14745/ccdr.v45i10a04f

Mots-clés : Relevé des maladies transmissibles au Canada, éditeur, maladies infectieuses
Cette oeuvre est mise à la disposition selon les termes de la licence internationale Creative Commons Attribution 4.0

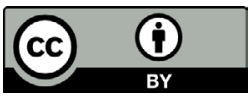

\section{Affiliation}

1 Bureau du conseiller scientifique en chef, Agence de la santé publique du Canada, Ottawa, ON

\section{*Correspondance : \\ phac.ccdr-rmtc.aspc@canada.ca}
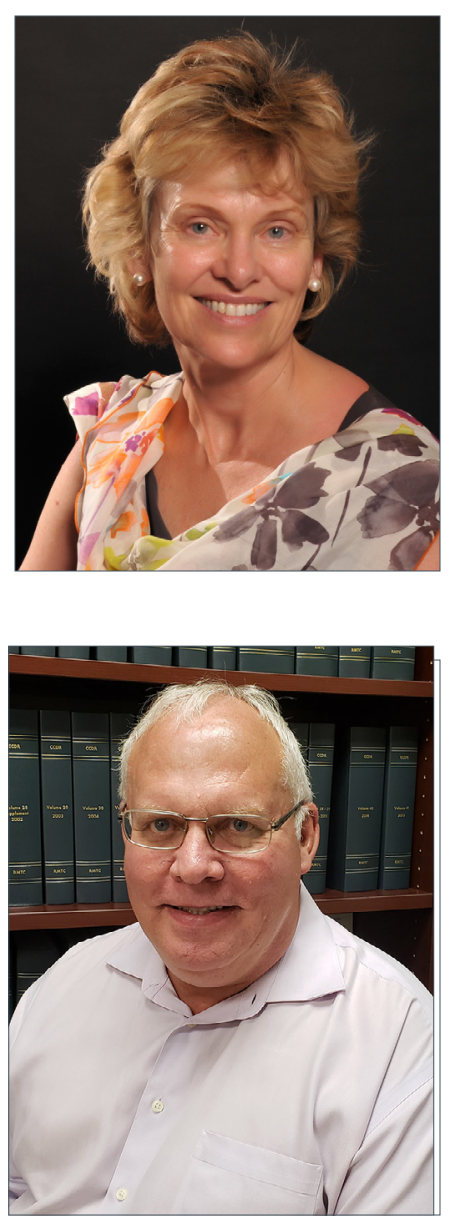